\title{
Implications of increased left ventricular mass index on in-hospital outcomes in patients undergoing aortic valve surgery
}

\author{
Rajendra H. Mehta, MD \\ David Bruckman, MS \\ Sugata Das, MD \\ Thomas Tsai, MD \\ Pamela Russman, BS \\ Dean Karavite, BS \\ Hillary Monaghan, RN \\ Seema Sonnad, PhD \\ Michael J. Shea, MD \\ Kim A. Eagle, MD \\ G. Michael Deeb, MD
}

From the Division of Cardiology and Section of Adult Cardiac Surgery (Heart Care Program), Division of General Pediatrics, University of Michigan, Ann Arbor, Mich.

Received for publication Sept 18, 2000; revisions requested Jan 3, 2001; revisions received April 17, 2001; accepted for publication April 19, 2001.

Address for reprints: G. Michael Deeb, MD, Professor of Surgery, Section of Adult Cardiac Surgery, 1500 E Medical Center Dr, Room 2124G/Box 0348, Ann Arbor, MI 48109-0348 (E-mail: mdeeb@umich.edu).

J Thorac Cardiovasc Surg 2001;122:919-28

Copyright (c) 2001 by The American Association for Thoracic Surgery

0022-5223/2001 $\$ 35.00+0 \quad \mathbf{1 2 / 1 / 1 1 6 5 5 8}$

doi: $10.1067 / \mathrm{mtc} .2001 .116558$
Background: Increased left ventricular mass index has been shown to be associated with higher mortality in epidemiologic studies. However, the effect of increased left ventricular mass index on outcomes in patients undergoing aortic valve replacement is unknown.

Methods: We studied 473 consecutive patients undergoing elective aortic valve replacement to assess the influence of left ventricular mass index on outcomes in patients having this procedure. Echocardiographic left ventricular dimensions were used to calculate left ventricular mass index (considered increased if $>134 \mathrm{~g} / \mathrm{m}^{2}$ in male patients and $>110 \mathrm{~g} / \mathrm{m}^{2}$ in female patients).

Results: Left ventricular mass index was increased in $24 \%$ of patients undergoing aortic valve replacement. Postprocedural complications (respiratory failure, renal insufficiency, congestive heart failure, and atrial and ventricular arrhythmias), length of stay in the intensive care unit, and in-hospital mortality were increased in patients with increased left ventricular mass index. Multivariable analysis identified prior valve surgery (odds ratio, $4.3 ; 95 \%$ confidence interval, 1.2-15.7; $P=.030$ ), left ventricular ejection fraction (odds ratio, 1.07; 95\% confidence interval, 1.01$1.14 ; P=.020$ ), history of hypertension (odds ratio, $8.2 ; 95 \%$ confidence interval, $2.2-30.4 ; P=.002$ ), history of liver disease (odds ratio, 50.4; $95 \%$ confidence interval, 4.2-609.0; $P=.002$ ), and increased left ventricular mass index (odds ratio, 38; 95\% confidence interval, 9.3-154.1; $P<.001$ ) as independent predictors of in-hospital mortality. Furthermore, low output syndrome was identified as the most common mode of death $(36 \%)$ after aortic valve replacement in patients with increased left ventricular mass index.

Conclusions: Increased left ventricular mass index is associated with increased adverse in-hospital clinical outcomes in patients undergoing aortic valve replacement. Although this finding warrants special modification in perioperative management, further studies are needed to address whether outcomes in asymptomatic patients with aortic valve disease could be improved by earlier aortic valve replacement before a significant increase in left ventricular mass index. 


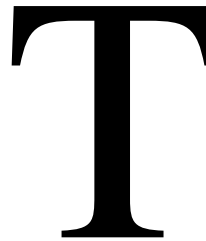

he chronically increased pressure-volume overload in patients with aortic valve disease results in left ventricular (LV) hypertrophy and altered geometry as an adaptive mechanism that helps maintain contractile performance despite markedly abnormal loading conditions. $^{1,2} \mathrm{LV}$ hypertrophy allows normalization of systolic wall stress and maintenance of ejection performance as afterload increases. ${ }^{1,2}$ Inadequate hypertrophy leads to afterload mismatch and can result in increased wall stress and decreased contractile performance. ${ }^{2}$ Extensive hypertrophy may result in depressed contractility and reduced pump function. ${ }^{3}$ Hypertrophy is associated with various LV diastolic abnormalities (including abnormal relaxation and distensibility $)^{4,5}$ and has been associated with increased morbidity and mortality in epidemiologic studies. ${ }^{6-9}$ The implications of increased LV mass on outcomes in patients undergoing aortic valve replacement (AVR) are poorly characterized. This study evaluates the influence of increased LV mass index (LVMI) on in-hospital outcomes of patients undergoing AVR.

\section{Methods \\ Study Population}

All patients undergoing elective AVR between January 1, 1992, and December 31, 1997, at the University of Michigan were prospectively identified through a routinely maintained surgical database. For the purpose of this analysis, patients were excluded if they underwent an AVR on an emergency basis, had poor acoustic windows for adequate echocardiographic assessment, and/or did not undergo an echocardiogram before the operation.

\section{Echocardiographic Methods}

All echocardiograms were performed within 3 months of AVR, as previously reported. ${ }^{10-12}$ End-diastolic measurements of LV internal dimension (LVID, in millimeters), interventricular septal thickness (IVST, in millimeters), and posterior wall thickness (PWT, in millimeters) were obtained according to the conventions established by the American Society of Echocardiography. ${ }^{11}$ Diastolic measurements were used to calculate LVMI by means of the formula described and validated by Devereux and colleagues ${ }^{10,12}$ as follows:

$$
\begin{gathered}
\text { LVMI }\left(\mathrm{g} / \mathrm{m}^{2}\right)=\left(1 . 0 4 \left[(\mathrm{IVST}+\mathrm{LVID}+\mathrm{PWT})^{3}-\right.\right. \\
\text { LVID } \left.\left.^{3}\right]-14 \mathrm{~g}\right) / \text { Body surface area }
\end{gathered}
$$

The LVMI was considered as increased if greater than $134 \mathrm{~g} / \mathrm{m}^{2}$ in men and greater than $110 \mathrm{~g} / \mathrm{m}^{2}$ in women. ${ }^{10-12}$

\section{Data Collection and Entry}

Medical charts were reviewed retrospectively on each patient to validate prior information and identify missing elements of interest. Data abstracted included patient demographics, symptoms, comorbidities, physical signs, medications, prior cardiac operations, predominant valvular lesion and cause, cardiac catheterization and echocardiographic data, and surgical data.

\section{Outcome Measures}

Chart review was also used to document in-hospital clinical events, length of stay, and in-hospital mortality for the study population.
Significant bleeding was defined as a need for transfusion of greater than 3 units, respiratory failure as a requirement for ventilator support for greater than 72 hours, renal insufficiency as an increase in baseline creatinine of greater than $2 \mathrm{mg} / \mathrm{dL}$ (in the absence of endstage renal failure on dialysis), and hepatic dysfunction as an elevation of hepatic enzymes of greater than 3 times normal levels. The modes of death were assigned as described previously. ${ }^{13}$

\section{Statistical Analysis}

Summary statistics are presented as frequencies and percentages, mean \pm standard deviation, or median and interquartile range. Associations among nominal variables were compared by $\chi^{2}$ tests, 2-sided Fisher exact tests, or Cochran-Mantel-Haenszel $\chi^{2}$ tests (for a trend within an ordinal category). Continuous univariate predictors for LVMI and death were tested by $t$ tests or WilcoxonMann-Whitney tests as appropriate.

For all logistic-regression modeling, a stepwise selection method was used initially to determine independent predictors of elevated LVMI and then to determine independent predictors of mortality. The initial findings were verified or modified by iterative modeling, testing interactions and quadratic effects. Models were compared by means of the likelihood ratio test and by the $R^{2}$ proportion reduction in likelihood ratio. The entire data set was used; no validation was performed, although diagnostic routines (eg, Hosmer and Lemeshow test for lack of fit, change in deviance and residuals, and leverage indicators) were used on final model selection. Odds ratios (ORs), $95 \%$ confidence intervals $(95 \% \mathrm{CI}), P$ values, and the approximate area under the receiver-operating curve, or c-statistic, were reported. Post hoc comparisons among the model variables were made with the standardized regression coefficient. This provided a method to rank the effect of variables across models.

\section{Propensity Analysis}

Because we imposed a dichotomy to LVMI, we sought to balance the effect of varying LVMI on the outcome of interest by developing propensity scores from a logistic model predicting normal or elevated LVMI. ${ }^{14-16}$ These scores reflect the probability of elevated LVMI for each patient, given a set of observed covariates. By stratifying the propensity scores into quintiles, the distributions of the background covariates predicting either normal or elevated LVMI should be similar. ${ }^{14-16}$

First, a predictive model for dichotomized LVMI was generated by using a set of variables suggestive of an association $(P<.20)$. Iterative modeling reduced the multivariate model (all $P<.05$ ). Interactions were tested iteratively and retained when suggestive of an association. This overparameterized model should help minimize the bias in estimating model coefficients and increase the efficiency in determining the model variance. ${ }^{16}$ The maximum number or parameters in the model to generate propensity scores was limited to $n^{1 / 2}$ or $\sqrt{ } 473=21 .{ }^{17}$ The predicted probabilities of elevated LVMI were ranked into quintiles, and the distributions of the model covariates were compared across LVMI groups. At least $95 \%$ of the comparisons showed no significant association to consider adequate balance within each stratum $(P>.05) .{ }^{18}$

Second, a predictive model for in-hospital mortality was generated by a set of variables suggestive of an association $(P<.20)$. A parsimonious model for mortality was developed. 


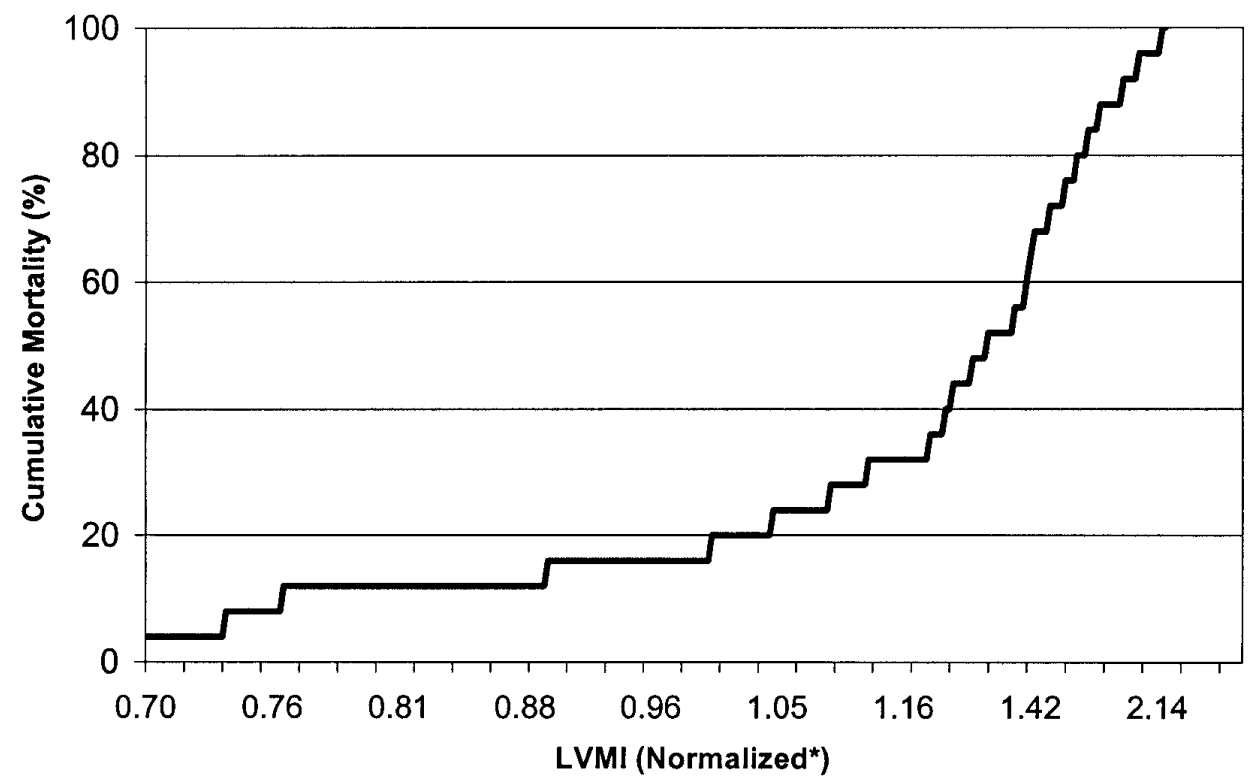

Figure 1. Cumulative mortality (\%) with increasing LVMI in patients undergoing elective AVR. *LVMI was normalized to adjust for sex differences by dividing LVMI by $110 \mathrm{~g} / \mathrm{m}^{2}$ in female patients and $134 \mathrm{~g} / \mathrm{m}^{2}$ in male patients.

Third, dummy variables indicating membership in 1 of the 5 quintiles of propensity scores were included in a new set of modeling iterations. The final iterations provided the model containing predictors of clinical relevance, statistical significance, or both. SAS (SAS Institute, Inc, Cary, NC) software was used for all analyses.

\section{Results}

\section{Study Population}

Of 509 patients who underwent elective AVR during the study period, 473 had echocardiographic data available and were included in this study (mean age, $61.2 \pm 14.5$ years; female patients, $61.3 \%$ ). Patients with and without increased LVMI were similar with respect to mean age, sex, and race distribution (Table 1). Baseline demographics and clinical symptoms and signs were similar, except for a higher proportion of patients with increased LVMI presenting with congestive heart failure and those with normal LVMI presenting with chest pain (Table 1). Comorbidities and medical and surgical histories were similar for the 2 groups of patients (Table 2).

\section{Echocardiographic LVMI Data}

Echocardiographic LVMI data are shown in Table 3. The mean LVMI for men and women were $107.5 \pm 45.1 \mathrm{~g} / \mathrm{m}^{2}$ and $94.6 \pm 40.9 \mathrm{~g} / \mathrm{m}^{2}$, respectively. The mean LVMI when adjusted for differences in sex by dividing LVMI by 134 $\mathrm{g} / \mathrm{m}^{2}$ in male patients and $110 \mathrm{~g} / \mathrm{m}^{2}$ in female patients were $0.86 \pm 0.37$ and $0.80 \pm 0.34$, respectively. An increased
LVMI was present in $24.3 \%$ of patients studied (22.4\% male patients and $27.3 \%$ female patients, $P=.23$ ).

\section{Surgical Data}

Surgical data are shown in Table 4. Isolated AVR was performed in $78.5 \%$ of patients, aortic and mitral valve replacement, repair, or both in $17.3 \%$, combined aortic arch replacement with AVR in $4.2 \%$, and concomitant coronary artery bypass surgery in $30 \%$. The predominant indication for AVR was aortic stenosis in 54\% and aortic regurgitation in $46 \%$ of patients. The mean ischemic time was similar for patients with or without elevated LVMI.

\section{Outcomes}

Clinical events, length of stay, and mortality are shown in Table 5. Compared with patients with normal LVMI, those with increased LVMI had a higher association of in-hospital congestive heart failure, cardiac arrest, bleeding requiring transfusion, respiratory failure, renal insufficiency, hepatic dysfunction, ventricular arrhythmias, need for inotropic support for greater than 48 hours, use of intra-aortic balloon pump, and sepsis. The incidence of atrial arrhythmias, mediastinitis, wound infection, stroke, and reexploration and the need for a permanent pacemaker were similar in the 2 groups of patients. Median length of stay in the intensive care unit was longer and in-hospital mortality was significantly higher for patients with increased LVMI than those without increased LVMI. The cumulative percentage of 
TABLE 1. Baseline characteristics

\begin{tabular}{|c|c|c|c|c|}
\hline Characteristics & Total & Increased LVMI & Normal LVMI & $P$ value \\
\hline No. & 473 & 115 & 358 & \\
\hline \multicolumn{5}{|l|}{ Patient demographics } \\
\hline Age, y (mean \pm SD [median]) & $61.2 \pm 14.5(64)$ & $61.2 \pm 14.7(63.8)$ & $61.2 \pm 14.5(64.0)$ & .99 \\
\hline Male, $\mathrm{n}(\%)$ & $290(61.3)$ & $65(56.5)$ & $225(62.8)$ & .23 \\
\hline White patients, n (\%) & $343(79.0)$ & $78(75.0)$ & $265(80.3)$ & .25 \\
\hline \multicolumn{5}{|l|}{ Clinical symptoms and signs } \\
\hline Syncope, n (\%) & $60(12.7)$ & $13(11.3)$ & $47(13.1)$ & .61 \\
\hline Angina, $\mathrm{n}(\%)$ & $41(8.7)$ & $9(7.8)$ & $32(8.9)$ & .71 \\
\hline Chest pain, $\mathrm{n}(\%)$ & $230(48.6)$ & $43(37.4)$ & $187(52.2)$ & .006 \\
\hline Congestive heart failure, $\mathrm{n}(\%)$ & $140(29.6)$ & $51(44.4)$ & $89(24.9)$ & $<.001$ \\
\hline New York Heart Association class $\geq 3$ & $232(68.3)$ & $88(76.5)$ & $235(65.6)$ & .029 \\
\hline Systolic blood pressure, mm Hg (mean \pm SD) & $131.1 \pm 34.1$ & $131.3 \pm 25.2$ & $131 \pm 36.5$ & .92 \\
\hline Diastolic blood pressure, $\mathrm{mm} \mathrm{Hg}$ (mean \pm SD) & $70.8 \pm 13$ & $66.8 \pm 13.3$ & $72 \pm 12.7$ & $<.001$ \\
\hline Elevated jugular venous pressure, $\mathrm{n}(\%)$ & $36(7.6)$ & $15(13.0)$ & $21(5.9)$ & .012 \\
\hline Bilateral rales $>$ one third of lung fields, $n(\%)$ & $40(8.5)$ & $15(13.0)$ & $25(7.0)$ & .042 \\
\hline Third heart sound, $\mathrm{n}(\%)$ & $64(13.5)$ & $28(24.4)$ & $36(10.1)$ & $<.001$ \\
\hline Pedal edema, $\mathrm{n}(\%)$ & $58(12.3)$ & $21(18.3)$ & $37(10.3)$ & .024 \\
\hline
\end{tabular}

TABLE 2. Comorbidities and past medical history

\begin{tabular}{|c|c|c|c|c|}
\hline Comorbidities & Total & Increased LVMI & Normal LVMI & $P$ value \\
\hline Hypertension, n (\%) & $196(41.4)$ & $49(42.6)$ & $147(41.1)$ & .77 \\
\hline Diabetic, $n(\%)$ & $63(13.3)$ & $13(11.3)$ & $50(14.0)$ & .46 \\
\hline Chronic obstructive pulmonary disease, $\mathrm{n}(\%)$ & $59(12.5)$ & $16(13.9)$ & $43(12)$ & .59 \\
\hline Coronary artery disease, $\mathrm{n}(\%)$ & $141(29.8)$ & $35(30.4)$ & $106(29.6)$ & .87 \\
\hline Elevated cholesterol, n (\%) & $72(15.2)$ & $10(8.7)$ & $62(17.3)$ & .025 \\
\hline Renal insufficiency, n (\%) & $19(4.0)$ & $9(7.8)$ & $10(2.8)$ & .017 \\
\hline Liver disease, $\mathrm{n}(\%)$ & $6(1.3)$ & $4(3.5)$ & $2(0.6)$ & .033 \\
\hline Peripheral vascular disease, $\mathrm{n}(\%)$ & $53(11.2)$ & $10(8.7)$ & $43(12.0)$ & .33 \\
\hline Current smokers, n (\%) & $131(27.7)$ & $33(28.7)$ & $98(27.4)$ & .78 \\
\hline \multicolumn{5}{|l|}{ Past medical history } \\
\hline Myocardial infarction, n (\%) & $43(9.1)$ & $12(10.4)$ & $31(8.7)$ & .56 \\
\hline Stroke, n (\%) & $50(10.6)$ & $10(8.7)$ & $40(11.2)$ & .45 \\
\hline Coronary angioplasty, n (\%) & $20(4.2)$ & $3(2.6)$ & $17(4.8)$ & .43 \\
\hline Coronary artery bypass surgery, $\mathrm{n}(\%)$ & $25(5.3)$ & $5(4.4)$ & $20(5.6)$ & .61 \\
\hline Valvular surgery, $\mathrm{n}(\%)$ & $48(10.2)$ & $16(13.9)$ & $32(8.9)$ & .12 \\
\hline Vascular surgery, $\mathrm{n}(\%)$ & $44(9.3)$ & $11(9.6)$ & $33(9.2)$ & .91 \\
\hline
\end{tabular}

mortality with increasing LVMI is shown in Figure 1. The proportion of patients who died increased with increasing deciles of LVMI (Table 6).

Univariate associations to mortality included edema at presentation, a history of gastrointestinal disease, carotid stenosis, a history of liver disease, alcoholism, a prior operation for peripheral vascular occlusive disease, prior valve surgery, history of hypertension, mitral stenosis, operation involving aortic arch reconstruction, catheterization, and increased LVMI (all $P<.05$ ). Various adverse events were associated with greater odds of in-hospital mortality: congestive heart failure, shock, cardiac arrest, pneumonia, mediastinitis, respiratory failure, renal failure, hepatic dysfunction, sepsis, cerebrovascular accident, transfusion requirement, ventilation, additional inotropic support, intra-aortic balloon support, or reexploration (all $P<.05$ ). Age greater than 65 years was marginally associated with mortality; however, this association did not trend linearly with increasing age. The 373 patients who had elective aortic valve surgery only had a lower mortality than all other valve operations or combinations of valve operations combined $(3.8 \%$ vs $11.0 \%, P<.004)$. Increased pump time was linearly associated with mortality $(P<.001)$.

In this phase of the analysis, we generated logistic models for mortality, adding LVMI initially and then the confounders 
TABLE 3. Echocardiographic data

\begin{tabular}{lcccc}
\hline Echocardiographic data & Total & Increased LVMI & Normal LVMI & \multicolumn{1}{c}{$\boldsymbol{P \text { value }}$} \\
\hline $\begin{array}{l}\text { Left ventricular ejection fraction, } \\
\quad \% \text { (mean [median]) }\end{array}$ & $52 \pm 11(56)$ & $46.2 \pm 13.6(50)$ & $54 \pm 9.7(60)$ & $<.001$ \\
Wall-motion abnormalities, $\%$ & $41(8.7)$ & $10(8.7)$ & $31(8.7)$ & .99 \\
LVMI, female patients (mean \pm SD) & $0.86 \pm 0.37$ & $1.35 \pm 0.30$ & $0.68 \pm 0.18$ & NA \\
LVMI, male patients (mean \pm SD) & $0.80 \pm 0.34$ & $1.30 \pm 0.29$ & $0.66 \pm 0.18$ & NA \\
\hline
\end{tabular}

$N A$, Not applicable.

*LVMI (in grams per square meters) was normalized to adjust for sex differences by dividing LVMI by $110 \mathrm{~g} / \mathrm{m}^{2}$ in female patients and $134 \mathrm{~g} / \mathrm{m}^{2}$ in male patients.

\section{TABLE 4. Surgical data}

\begin{tabular}{|c|c|c|c|c|}
\hline Predominant valvular lesion & Total & Increased LVMI & Normal LVMI & $P$ value \\
\hline Aortic stenosis, n (\%) & $255(53.9)$ & $48(41.7)$ & $207(57.8)$ & .003 \\
\hline Aortic regurgitation, $\mathrm{n}(\%)$ & $214(45.2)$ & $67(58.3)$ & $147(41.1)$ & $<.001$ \\
\hline \multicolumn{5}{|l|}{ Other surgical data } \\
\hline Mitral valve surgery, n (\%) & $82(17.3)$ & $28(24.4)$ & $54(15.1)$ & .022 \\
\hline Coronary artery bypass surgery, $\mathrm{n}(\%)$ & $161(34.0)$ & $42(36.5)$ & $119(33.2)$ & .52 \\
\hline Aortic arch replacement, $\mathrm{n}(\%)$ & $20(4.2)$ & $5(4.4)$ & $15(4.2)$ & .94 \\
\hline Tricuspid valve repair, n (\%) & $17(3.6)$ & $5(4.4)$ & $12(3.4)$ & .62 \\
\hline $\begin{array}{l}\text { Operative ischemic time, } \\
\min (\text { mean } \pm S D \text { [median]) }\end{array}$ & $144 \pm 59(140)$ & $150 \pm 52(142)$ & $142 \pm 61(138)$ & .18 \\
\hline
\end{tabular}

of LVMI to the predictive model. Several of these modifiers were already in the original model because of an association with mortality. The additional variables were pulmonary edema, third heart sound, presence of aortic regurgitation or aortic stenosis as predominant lesions, and pulse pressure.

The logistic-regression model for mortality including LVMI revealed a list of variables associated with both mortality and increased LVMI (Table 7). The associations to mortality were as follows: LVMI (OR, 23.1; 95\% CI, 7.2$73.9 ; P<.001)$; history of liver disease (OR, $36.3 ; 95 \% \mathrm{CI}$, $4.2-314.3 ; P=.001)$; age greater than 65 years in main and quadratic terms (OR, $0.80 ; 95 \% \mathrm{CI}, 0.64-1.00 ; P=.052$; and OR, 1.002; 95\% CI, 1.000-1.004; $P=.041$, respectively); prior valve surgery (OR, 3.47; 95\% CI, 1.04-11.63; $P<$ .043 ); history of hypertension (OR, 5.91; 95\% CI, 1.7520.02; $P=.004)$; and carotid stenosis (OR, $5.95 ; 95 \% \mathrm{CI}$, $1.42-24.91 ; P<.014)$. The fit of the model was $\operatorname{good}(P=$ .82 , c-statistic $=0.90$ ) where the predicted number of deaths had high correspondence with the actual deaths across the continuum of risk (data not shown).

\section{Propensity Analysis}

Propensity scores were generated by means of a logisticregression model for elevated LVMI to derive more robust model estimates of variance. This model included 13 confounding variables seen with strong univariate associates $(P<$ $.05)$, including categoric interactions. Propensity scores were ranked and classified into quintiles. Within each quintile, cross-tabulations and Student $t$ tests were run across increased and normal LVMI classes. Although 13 of the 65 comparisons had too few counts to compare across classes, only one of the remaining 52 comparisons failed to balance on the observed covariates (with $P>.05$ ). This indicated that the probability of elevated or normal LVMI was relatively similar within a quintiled stratum, conditional on the observed covariates.

Last, we regenerated the logistic regression model for mortality with dummy variables that reflected membership into each quintile of propensity score. The main effect of age and the quadratic age, in addition to an indication of carotid stenosis, were removed from the model. The final model retained all other covariates, including LVMI and LV ejection fraction (Table 8).

After adjustment, this model identified the following associations to an increased risk of in-hospital mortality: an increased LVMI (OR, 37.9; 95\% CI, 9.3-154.1; $P<.001)$; a history of liver disease (OR, 50.4; 95\% CI, 4.2-609.0; $P=$ .001 ); a history of hypertension (OR, 8.2; 95\% CI, 2.2-30.4; $P=.002)$; LV ejection fraction (OR, 1.07; 95\% CI, 1.01-1.14; $P=.020)$; and prior valve surgery $(\mathrm{OR}, 4.3 ; 95 \% \mathrm{CI}, 1.2-$ $15.7 ; P=.03)$. Increased LVMI remained the strongest predictor of mortality, having more than twice the standardized estimate than all other covariates (eg, 0.858 vs 0.436 for LV ejection fraction). Confounders did not reduce or modify greatly the predictive strength of LVMI. The final model provided an excellent predictive fit $(P=.14, d f=8 ;$ c-statistic $=$ $0.90)$. 
TABLE 5. Outcomes

\begin{tabular}{|c|c|c|c|c|}
\hline Clinical events & Total & Increased LVMI & Normal LVMI & $P$ value \\
\hline Congestive heart failure, $\mathrm{n}(\%)$ & $17(3.6)$ & $10(8.7)$ & $7(2.0)$ & .002 \\
\hline Cardiac arrest, $\mathrm{n}(\%)$ & $18(3.8)$ & $14(12.2)$ & $4(1.1)$ & $<.001$ \\
\hline Bleeding, $\mathrm{n}(\%)$ & $50(10.6)$ & $20(17.4)$ & $30(8.4)$ & .006 \\
\hline Atrial fibrillation, $\mathrm{n}(\%)$ & $165(34.9)$ & $49(42.6)$ & $116(32.4)$ & .046 \\
\hline Mediastinitis, $\mathrm{n}(\%)$ & $9(1.9)$ & $2(1.7)$ & $7(2.0)$ & .99 \\
\hline Ventricular arrhythmias, $\mathrm{n}(\%)$ & $28(5.9)$ & $15(13)$ & $13(3.6)$ & $<.001$ \\
\hline Renal failure, $\mathrm{n}(\%)$ & $28(5.9)$ & $17(14.8)$ & $11(3.1)$ & $<.001$ \\
\hline Respiratory failure, n (\%) & $37(7.8)$ & $22(19.1)$ & $15(4.2)$ & $<.001$ \\
\hline Hepatic dysfunction, n (\%) & $10(2.1)$ & $7(6.1)$ & $3(0.8)$ & .003 \\
\hline Inotropic support >48 hours, n (\%) & $24(5.1)$ & $17(14.8)$ & $7(2.0)$ & $<.001$ \\
\hline Intra-aortic balloon counterpulsation, $\mathrm{n}(\%)$ & $10(2.1)$ & $8(7.0)$ & $2(0.6)$ & $<.001$ \\
\hline Sepsis, n (\%) & $22(4.6)$ & $10(8.7)$ & $12(3.4)$ & .018 \\
\hline Stroke, $\mathrm{n}(\%)$ & $26(5.5)$ & $8(7.0)$ & $18(5.0)$ & .43 \\
\hline Permanent pacemaker, $\mathrm{n}(\%)$ & $21(4.4)$ & $8(7.0)$ & $13(3.6)$ & .13 \\
\hline Reexploration, n (\%) & $13(2.7)$ & $5(4.3)$ & $8(2.2)$ & .23 \\
\hline Length of ICU stay, $d$ (median [IQR])* & $2(1-3)$ & $3(2-8)$ & $2(1-3)$ & $<.001^{*}$ \\
\hline In-hospital mortality, n (\%) & $25(5.3)$ & $20(17.4)$ & $5(1.4)$ & $<.001$ \\
\hline
\end{tabular}

ICU, Intensive care unit.

${ }^{*}$ Comparison made on medians, distribution by using Wilcoxon rank sum test.

TABLE 6. Distribution of survivors and mortalities across levels of LVMI

\begin{tabular}{lccc}
\hline LVMI midpoint & Survivors & Mortalities & $\begin{array}{c}\text { Percentage } \\
\text { of mortality }\end{array}$ \\
\hline 0.2 & 8 & 1 & 12.5 \\
0.4 & 61 & 0 & 0 \\
0.6 & 132 & 0 & 0 \\
0.8 & 109 & 3 & 2.8 \\
1.0 & 69 & 2 & 2.9 \\
1.2 & 36 & 7 & 19.4 \\
1.4 & 13 & 5 & 38.5 \\
1.6 & 9 & 4 & 44.4 \\
1.8 & 7 & 1 & 14.3 \\
2.0 & 4 & 2 & 50.0 \\
Sum & 448 & 25 & \\
\hline
\end{tabular}

Age and sex dropped out early in model selection. The sex adjustment inherent in LVMI and the age adjustment in propensity scores may have accounted for the lack of statistical significance. Mean LV ejection fraction did not differ between surviving and dead patients, yet it was significant in predicting death. Other strong effects of confounders to LVMI, such as congestive heart failure, New York Heart Association class, third heart sound, predominant aortic regurgitation, or stenosis, were removed during modeling.

Forty-eight percent of patients with hospital death had at least 2 of 4 nominal factors (history of liver disease, history of hypertension, prior valve surgery, and increased LVMI) versus $4.9 \%$ of survivors $(P<.001)$. In testing whether having at least 2 of 4 factors was associated with mortality,
94.6\% specificity and $35.3 \%$ sensitivity was obtained in predicting in-hospital death after AVR. The probability that an AVR patient with less than 2 nominal factors survives the operation (ie, negative predictive value) was $91.1 \%$.

Among the 25 patients who died after AVR, modes of death are as shown in Table 9. Low output syndrome was determined as the cause of death in 9 of 25 patients, whereas 16 patients died of causes other than low output syndrome. The LVMI was increased in all 9 patients who died of low output syndrome $(P=.12)$, whereas no patient with normal LVMI died of a low cardiac output.

\section{Discussion}

Our study identified an increased LVMI as a strong predictor of poor outcomes after elective AVR, specifically increased in-hospital clinical events, length of stay, and inhospital mortality. We found LVMI, as a predictor of mortality, to be highly resistant to confounding by variables that were directly associated with increased LVMI. Furthermore, we identified low cardiac output as the most common mode of death in patients with increased LVMI undergoing AVR.

Previous studies have identified factors that increase operative morbidity and mortality in patients undergoing AVR, including advanced age, prior AVR, associated coronary artery disease, advanced functional class, LV dysfunction, aortic regurgitation as opposed to aortic stenosis, atrial fibrillation, and renal insufficiency. ${ }^{19-21}$ Epidemiologic studies ${ }^{6-9}$ and studies in patients with hypertension, ${ }^{22}$ have identified increased LVMI as an important marker for increased morbidity and mortality. Our findings confirm the association of poor outcomes with increased LVMI in patients undergoing AVR. 
TABLE 7. Univariate associations with mortality and model when modeling with variables associated with mortality and including LVMI and its confounders among patients undergoing elective aortic valve surgery

\begin{tabular}{|c|c|c|c|c|c|c|}
\hline \multirow[b]{2}{*}{ Variable } & \multicolumn{3}{|c|}{ Univariate } & \multicolumn{3}{|c|}{ Logistic model } \\
\hline & Overall (of 473) & Deaths (of 25) & $P$ value & $\mathbf{O R}$ & $95 \% \mathrm{Cl}$ & $P$ value \\
\hline Increased LVMI (>1) & $24.3 \%$ & $80.0 \%$ & $<.001$ & 23.1 & $7.2-73.9$ & $<.001$ \\
\hline History of liver disease & $1.3 \%$ & $12.0 \%$ & $<.001$ & 36.3 & $4.2-314.3$ & .001 \\
\hline Age, y (mean \pm SD) & $61.2 \pm 14.5$ & $62.3 \pm 16.6$ & .73 & 0.80 & $0.64-1.00$ & .052 \\
\hline Quadratic age $>65 y$ & $3955 \pm 1674$ & $4151 \pm 1836$ & .59 & 1.002 & $1.000-1.004$ & .041 \\
\hline Prior valve surgery & $10.1 \%$ & $28.0 \%$ & .002 & 3.5 & $1.04-11.6$ & .044 \\
\hline LV ejection fraction (mean \pm SD) & $52.1 \pm 11.3$ & $52.3 \pm 9.9$ & .92 & 1.06 & $1.01-1.11$ & .024 \\
\hline Carotid stenosis & $6.8 \%$ & $16.0 \%$ & .06 & 6.0 & $1.4-24.9$ & .014 \\
\hline History of hypertension & $11.4 \%$ & $24.0 \%$ & .042 & 5.9 & $1.7-20.0$ & .004 \\
\hline \multicolumn{4}{|l|}{ Intercept: parameter coefficient (SE) } & \multicolumn{3}{|c|}{$-2.9(3.19)$} \\
\hline
\end{tabular}

$O R$, Odds ratio; $C l$, confidence interval.

Our finding is similar to that of the study of Orsinelli and colleagues, ${ }^{23}$ who showed that LV hypertrophy (increased relative LV wall thickness) was associated with greater postoperative mortality after AVR for aortic stenosis. However, several differences between our study and that of Orsinelli and colleagues deserve comment. First, we included all patients undergoing AVR for a variety of conditions, not just isolated aortic stenosis. Second, we used LVMI, not relative LV wall thickness, as a measure of LV hypertrophy. Third, beyond the increased in-hospital mortality, we showed increased nonfatal events and length of stay among patients with an increased LVMI. Finally, we identified low output syndrome as the most common mode of death.

Several mechanisms may operate in the elevated risk accompanying an increased LVMI in patients having AVR. Excessive LV hypertrophy has been shown to be associated with contractile impairment and pump dysfunction, leading to congestive heart failure. ${ }^{3,8}$ Furthermore, heterogeneity in diastolic function has been reported in patients with aortic stenosis, varying from abnormal relaxation to reduced distensibility. ${ }^{4,5,24-27}$ This effect is similar to that seen in pressure-overload hypertrophy in patients with hypertension. ${ }^{28}$ Mechanisms underlying this heterogeneity include individual differences in the severity of muscle hypertrophy, systolic dysfunction, or both or differences in the severity of mitral regurgitation and in the level of left atrial pressure. ${ }^{24,25,28}$ Echocardiographic data on measures of diastolic function were not available in our patients, and on average, systolic function was decreased in patients with increased LVMI compared with in those without increased LVMI (Table 3). Multivariable modeling, controlling for systolic dysfunction, showed that increased LVMI remained a highly significant predictor of in-hospital mortality. Furthermore, the mean LV ejection fraction was relatively preserved $(52 \% \pm 11 \%)$ in our study and was directly associated with increased mortality in the multivariate model.
TABLE 8. Final logistic-regression model for mortality adjusted with propensity scores for the probability of elevated LVMI conditional on observed covariates associated with LVMI among patients undergoing elective aortic valve surgery

\begin{tabular}{lccc}
\hline Variable & Odds ratio & 95\% Cl & Pvalue \\
\hline LVMI (mean \pm SD) & 37.9 & $9.3-154.1$ & $<.001$ \\
History of liver disease & 50.4 & $4.2-609.0$ & .001 \\
History of hypertension & 8.2 & $2.2-30.4$ & .002 \\
LV ejection fraction & 1.07 & $1.01-1.14$ & .020 \\
$\quad$ (mean \pm SD) & & & \\
Prior valve surgery & 4.3 & $1.2-15.7$ & .030 \\
Intercept (parameter & $-9.65(2.22)$ & & \\
$\quad$ coefficient [SE]) & & & \\
\hline
\end{tabular}

Results for the dummy variables reflecting propensity score; strata are not shown.

This suggests that in patients with normal or near-normal LV ejection fraction, the greater degree of diastolic dysfunction caused by greater contractility and smaller hearts may worsen outcomes in such patients. Similarly, systolic hypertension remained an important independent predictor of mortality in the multivariate model. A possible explanation could be that for a given degree of LV hypertrophy, hypertensive patients may have greater diastolic dysfunction than those without elevated systolic blood pressure.

Aurigemma and colleagues, ${ }^{29}$ in a study with a small number of patients undergoing AVR for aortic stenosis, showed that a subset of these patients has an abnormal intracavitary flow acceleration postoperatively that is often associated with distinct LV geometry (small cavity with marked hypertrophy) and supernormal LV function. This subset had systemic hypotension and a low cardiac output postoperatively, despite normal LV ejection fraction. This occurred in 
TABLE 9. Relationship of mode of death to increased LVMI

\begin{tabular}{lccc}
\hline & \multicolumn{3}{c}{ LVMI } \\
\cline { 2 - 4 } Death & Normal & Increased & Total \\
\hline Low output syndrome & 0 & 9 & 9 \\
Other causes* & 5 & 11 & 16 \\
Total & 5 & 20 & 25 \\
\hline
\end{tabular}

*Bleeding from coagulopathy (5); recurrent supraventricular and ventricular tachycardia (3); sepsis caused by mediastinitis-wound infection (2); thyroid storm caused by amiodarone (1); intracranial bleed (cerebral aneurysm [1], spontaneous [1]); occipitoparietal infarction (1); spontaneous dehiscence of distal aortic graft (1); and acute on chronic liver failure, sepsis, and renal shutdown (1).

the absence of systolic anterior motion of the mitral valve leaflet, asymmetric septal hypertrophy causing outflow obstruction, or both. The authors speculated that the drop in LV afterload after AVR and concomitant treatment with positive inotropic agents enhanced intracavitary flow velocity and exacerbated abnormal ejection dynamics. In our study the most common mode of death was low output syndrome $(36 \%)$, accounting for half of the deaths in patients with increased LVMI. No patients with a normal LVMI died of a low cardiac output syndrome. Inotropic drugs and intra-aortic balloon counterpulsation were more frequently used in the postoperative period in patients with increased LVMI. The resultant increase in contractility and reduction in afterload could have contributed to abnormal LV ejection dynamics and poor outcomes, as observed in the study by Aurigemma and colleagues.

Some investigators have reported an increase in mortality when patients require coronary artery bypass surgery in addition to AVR compared with that seen in patients requiring AVR alone. In our study the need for concurrent coronary artery bypass surgery was similar in patients with and without increased LVMI and thus could not have accounted for the difference in outcomes observed. Abnormalities of coronary flow reserve have been reported in patients with increased LVMI in the absence of flow-limiting coronary artery stenosis. ${ }^{30,31}$ Marcus and colleagues ${ }^{31}$ showed a reduction in coronary blood flow velocity during reactive hyperemia at the time of operation in patients with severe aortic stenosis. This aberrance of flow reserve aggravates ischemia of the left ventricle in addition to that caused by frequent association of aortic valve disease with coronary artery disease and the supply and demand mismatch unique to this disease process and leads to systolic and diastolic LV dysfunction in the perioperative period. ${ }^{32-34}$ Thus, the hypertrophied left ventricle poses a special problem for effective cardioprotection during ischemic arrest, even in a beating heart on extracorporeal circulation, where the mean perfusion pressures are lower, as observed during low blood pressure in hypertensive patients who have a hypertrophied heart. ${ }^{35}$
LV hypertrophy predisposes to the development of cardiac arrhythmias ${ }^{36-38}$ and sudden death. ${ }^{9}$ The propensity for rhythm disturbances may be exaggerated in the perioperative period as a result of alterations in the metabolic parameters and the use of drugs that could potentiate arrhythmias. We observed an increase in serious ventricular arrhythmias and a trend toward more atrial arrhythmias in patients with increased LVMI.

Finally, 2 other factors were identified to be associated with increased in-hospital mortality in patients undergoing AVR: repeat valve surgery and liver disease. Prior studies have shown increased mortality with repeat AVR, and our finding is consistent with the results of these studies. ${ }^{19-21}$ The association of liver disease with increased mortality may be related to associated coagulopathy, increased risk of infection, volume overload, renal insufficiencies, or overall poor health status of such patients.

\section{Clinical Implications}

This study suggests that patients undergoing elective AVR who have increased LVMI should have all precautions taken during ischemic arrest to optimize cardioprotection. Adequate cardioplegic solution should be delivered for preservation of the entire myocardium, and retrograde administration through coronary sinus infusion should be considered. Coronary artery disease should be aggressively treated to prevent ischemia in the perioperative period. A decrease in afterload caused by intra-aortic balloon counterpulsation may worsen the hemodynamic profile of patients with critical aortic stenosis. Thus, its routine use cannot be recommended for patients undergoing AVR. Careful monitoring for metabolic parameters should be performed, and any abnormalities should be promptly corrected to prevent serious arrhythmias. Hypotension should be expeditiously reversed with volume resuscitation and use of $\alpha$-agonists rather than positive inotropic agents. Congestive heart failure from diastolic dysfunction should be treated by slowing the heart rate (calcium channel and $\beta$-blockers) or converting tachyarrhythmias as appropriate. All such precautions in patients with increased LVMI undergoing AVR may help improve their outcomes. Finally, as noted previously, our findings raise an important issue as to whether patients with severe aortic valve disease should have AVR earlier in the course of their disease, when they are minimally symptomatic or even asymptomatic and before the development of more severe LV hypertrophy leads to a greater perioperative risk. ${ }^{39}$

\section{Limitations of the Study}

Although patients were prospectively identified, much of the data were obtained by means of a chart review, and thus the analysis has limitations inherent to such studies. Also, although we identified attributes and outcomes associated with increased LVMI, causality cannot be proven. Most AVRs 
at our center ( $>95 \%)$ were performed by a single surgeon specializing in aortic operations. Thus, our findings may not be generalizable to other centers or surgeons that perform few AVRs. Our results are not applicable for patients undergoing emergency AVRs because we excluded them. The LVMI was estimated by means of M-mode echocardiography, which may not be valid in the setting of abnormal LV geometry. Also, the long-term effects of LVMI on outcomes after AVR cannot be determined from this study. Finally, although no validation was performed in our study, the consistent results in modeling with and without adjustment by use of a multivariate model and propensity scores reveals the predictive strength of LVMI to mortality, conditional on observed covariates. Thus, we believe that analyses at other institutions or from a regional or national database will validate that LVMI is an exceptionally important predictor of mortality.

\section{Conclusion}

Increased LVMI is associated with increased in-hospital clinical events, greater length of stay, and increased in-hospital mortality in patients undergoing AVR. This finding calls for special attention to perioperative management of such patients. Whether asymptomatic patients with significant aortic valve disease and an increased LVMI would benefit from earlier AVR requires further investigation.

\section{References}

1. Spann JF, Bove AA, Natrajan G, Kreulens T. Ventricular performance, pump function, and compensatory mechanisms in patients with aortic stenosis. Circulation. 1980;62:576-82.

2. Ross J. Afterload mismatch in aortic and mitral valve disease: implications for surgical therapy. J Am Coll Cardiol. 1985;5:811-26.

3. Wisenbaugh T, Booth D, DeMaria, A, Nissen S, Waters J. Relationship of contractile state to ejection performance in patients with chronic aortic valve disease. Circulation. 1986;73:47-53.

4. Eichhorn P, Grimm J, Koch R, Hess O, Carroll J, Krayenbuehl HP. Left ventricular relaxation in patients with left ventricular hypertrophy secondary to aortic valve disease. Circulation. 1982;65:1395-404.

5. Grossman W, McLaurin LP, Stefandouros MA. Left ventricular stiffness associated with chronic pressure and volume overloads in man. Circ Res. 1974;35:793-800.

6. Levy D, Garrison RJ, Savage DD, Kannel WB, Castelli WP. Prognostic implications of echocardiographically determined left ventricular mass in Framingham Heart Study. $N$ Engl $J$ Med. 1990;323:1561-6.

7. Galderisi L, Lauer MS, Levy D. Echocardiographic determinants of clinical outcome in subjects with coronary artery disease (the Framingham Heart Study). Am J Cardiol. 1992;72:971-6.

8. Vasan RS, Levy D. The role of hypertension in the pathogenesis of heart failure: a clinical mechanistic overview. Arch Intern Med. 1996; 156:1789-96.

9. Haider AW, Larson MG, Benjamin EJ, Levy D. Increased left ventricular mass and hypertrophy are associated with increased risk for sudden death. J Am Coll Cardiol. 1998;32:1454-59.

10. Devereux RB, Reichek N. Echocardiographic determination of LV mass in man: anatomic validation of the method. Circulation. 1977; 55:613-9.

11. Sahn DJ, Demaria A, Kisslo J, Weyman A. Recommendations regarding quantitation in M-mode echocardiography: results of survey of echocardiographic measurements. Circulation. 1978;58:1072-81.

12. Devereux RB, Alonso DR, Lutas EM, Gottlieb GJ, Campo E, Sachs I, et al. Echocardiographic assessment of left ventricular hypertrophy: comparison to necropsy findings. Am J Cardiol. 1986;57:450-8.

13. O'Connor GT, Birkmeyer JD, Dacey LJ, Quinton HB, Marrin CA, Birkmeyer NJ,et al. Results of a regional study of modes of death associated with coronary artery bypass grafting. Northern New England Cardiovascular Disease Study Group. Ann Thorac Surg. 1998; 66:1323-8.

14. Rosenbaum PR, Rubin DB. Reducing bias in observational studies using subclassification on the propensity score. J Am Stat Assoc. 1984;79:516-23.

15. Rosenbaum PR, Rubin DB. The central role of the propensity score in observational studies for causal effects. Biomerika. 1983;70:41-55.

16. Joffe MM, Rosenbaum PR. Invited commentary: propensity scores. Am J Epidemiol. 1999;150:327-33.

17. Robins JM, Mark SD, Newey WK. Estimating exposure effects by modeling the expectation of exposure conditional in confounders. Biometrics. 1992;48:479-95.

18. Cochran WG. The effectiveness of adjustment by subclassification in removing bias in observational studies. Biometrics. 1968;24:295-313.

19. Lytle BW, Cosgrove DM, Taylor PC, Goormastic M, Stewart RW, Golding LA, et al. Primary isolated aortic valve replacement. J Thorac Cardiovasc Surg. 1989;97:675-94.

20. Craver JM, Weintraub WS, Jones EL, Guyton RA, Hatcher CR. Predictors of mortality, complications, and length of stay in aortic valve replacement for aortic stenosis. Circulation. 1988;78(Suppl):I-85-90.

21. Lund O. Preoperative risk evaluation and stratification of long-term survival after valve replacement for aortic stenosis. Circulation. 1990; 82:124-39.

22. Casale PN, Devereux RB, Milner M, Zullo G, Harshfield GA, Pickering TG, et al. Value of echocardiographic left ventricular mass in predicting cardiac morbid events in hypertensive men. Ann Intern Med. 1986;105:173-8.

23. Orsinelli DA, Aurigemma GP, Battista S, Krendel S, Gaasch WH. Left ventricular hypertrophy and mortality after aortic valve replacement for aortic stenosis: a high risk subgroup identified by preoperative relative wall thickness. J Am Coll Cardiol. 1993;22:1679-83.

24. Lavine SJ, Follansbee WP, Shreiner DP, Amidi M. Left ventricular diastolic filling in valvular aortic stenosis. Am J Cardiol. 1986; 57:1349-55.

25. Murakami T, Hess OM, Gage JE, Grimm J, Krayenbuehl HP. Diastolic filling dynamics in patients with aortic stenosis. Circulation. 1986; 73:1162-74

26. Otto CM, Pearlman AS, Amsler LC. Doppler echocardiographic evaluation of left ventricular diastolic filling in isolated valvular aortic stenosis. Am J Cardiol. 1989;63:313-6.

27. Vanoverschelde JLJ, Essamri B, Michel X, Hanet C, Cosyns JR, Detry $\mathrm{JM}$, et al. Hemodynamic and volume correlates of left ventricular diastolic relaxation and filling in patients with aortic stenosis. J Am Coll Cardiol. 1992;20:813-21.

28. Hartford M, Wikstrand J, Wallentin I, Ljungman S, Wilhelmsen L, Berglund G. Diastolic function of heart in untreated primary hypertension. Hypertension. 1984;6:329-38.

29. Aurigemma G, Battista S, Orsinelli S, Sweeney A, Pape L, Cuenoud $\mathrm{H}$. Abnormal left ventricular intracavitary flow acceleration in patients undergoing aortic valve replacement for aortic stenosis: a marker for high post-operative morbidity and mortality. Circulation. 1992;86: 926-36.

30. Polese A, De Cesare N, Montorsi P, Fabbiocchi F, Guazzi M, Loaldi A, et al. Upward shift of the lower range of coronary flow autoregulation in hypertrophy of the left ventricle. Circulation. 1991;83:845-53.

31. Marcus ML, Dot DB, Hiratzka LF, Wright CB, Eastham CL. Decreased coronary flow reserve: a mechanism for angina pectoris in patients with aortic stenosis and normal coronary arteries. $N$ Engl $J$ Med. 1982;307:1362-6.

32. Vinten-Johansen J, Weiss HR. Oxygen consumption in subepicardial and subendocardial regions of canine left ventricle: the effect of experimental acute valvular stenosis. Circ Res. 1980;46:139-45.

33. Smucker ML, Tedesco CL, Manning SB, Owen RM, Feldman MD. Demonstration of an imbalance between coronary perfusion and 
excessive load as a mechanism of ischemia during stress in patients with aortic stenosis. Circulation. 1988;78:573-82.

34. Matsuo S, Tsuruta M, Hayano M, Imamura Y, Eguchi Y, Tokushima T, et al. Phasic coronary flow velocity determined by Doppler flow catheter in aortic stenosis and aortic regurgitation. Am J Cardiol. 1988;62:917-22.

35. Mansour P, Bostrom PA, Mattiasson I, Lilja B, Berglund G. Low blood pressure levels and signs of myocardial ischemia: importance of left ventricular hypertrophy. J Hum Hypertens. 1993;7:13-8.

36. Kannel WB, Gordon T, Offutt D. Left ventricular hypertrophy by elec- trocardiogram: prevalence, incidence and mortality in the Framingham Study. Ann Intern Med. 1969;71:89-105.

37. McLenachan JM, Henderson E, Morris KI, Dargie HJ. Ventricular arrhythmias in hypertensive left ventricular hypertrophy. $N$ Engl J Med. 1987;317:787-92.

38. Levy D, Anderson KM, Savage DD, Balkus SA, Kannel WB, Castelli WP. Risk of ventricular arrhythmias in left ventricular hypertrophy: the Framingham Heart Study. Am J Cardiol. 1987;60:560-5.

39. Lund O. The curative potential of early operation. Scand J Thorac Cardiovasc Surg (Suppl). 1993;40:113-22.

\section{ON THE MOVE?}

Send us your new address at least six weeks ahead

Don't miss a single issue of the journal! To ensure prompt service when you change your address, please photocopy and complete the form below.

Please send your change of address notification at least six weeks before your move to ensure continued service. We regret we cannot guarantee replacement of issues missed due to late notification.

\section{JOURNAL TITLE:}

Fill in the title of the journal here.

\section{OLD ADDRESS:}

Affix the address label from a recent issue of the journal here.

\section{NEW ADDRESS:}

Clearly print your new address here.

Name

Address

City/State/ZIP
COPY AND MAIL THIS FORM TO:

Mosby

Subscription Customer Service

6277 Sea Harbor Dr

Orlando, FL 32887
OR FAX TO:

407-363-9661

N/M Mosby
OR PHONE:

800-654-2452

Outside the U.S., call

407-345-4000 